\title{
Real-time ultrasound-guided endoscopic surgery for putaminal hemorrhage
}

\author{
Hirokazu Sadahiro, MD, Sadahiro Nomura, MD, Hisaharu Goto, MD, Kazutaka Sugimoto, MD, \\ Akinori Inamura, MD, Yuichi Fujiyama, MD, Akiko Yamane, MD, Takayuki Oku, MD, \\ Mizuya Shinoyama, MD, and Michiyasu Suzuki, MD
}

Department of Neurosurgery and Clinical Neuroscience, Yamaguchi University School of Medicine, Ube, Yamaguchi, Japan

OBJECT Endoscopic surgery plays a significant role in the treatment of intracerebral hemorrhage. However, the residual hematoma cannot be measured intraoperatively from the endoscopic view, and it is difficult to determine the precise location of the endoscope within the hematoma cavity. The authors attempted to develop real-time ultrasound-guided endoscopic surgery using a bur-hole-type probe.

METHODS From November 2012 to March 2014, patients with hypertensive putaminal hemorrhage who underwent endoscopic hematoma removal were enrolled in this study. Real-time ultrasound guidance was performed with a bur-holetype probe that was advanced via a second bur hole, which was placed in the temporal region. Ultrasound was used to guide insertion of the endoscope sheath as well as to provide information regarding the location of the hematoma during surgical evacuation. Finally, the cavity was irrigated with artificial cerebrospinal fluid and was observed as a low-echoic space, which facilitated detection of residual hematoma.

RESULTS Ten patients with putaminal hemorrhage $>30 \mathrm{~cm}^{3}$ were included in this study. Their mean age $( \pm$ SD) was $60.9 \pm 8.6$ years, and the mean preoperative hematoma volume was $65.2 \pm 37.1 \mathrm{~cm}^{3}$. The mean percentage of hematoma that was evacuated was $96 \% \pm 3 \%$. None of the patients exhibited rebleeding after surgery.

CONCLUSIONS This navigation method was effective in demonstrating both the real-time location of the endoscope and real-time viewing of the residual hematoma. Use of ultrasound guidance minimized the occurrence of brain injury due to hematoma evacuation.

http://thejns.org/doi/abs/10.3171/2014.11.JNS141508

KEY WORDS intracerebral hemorrhage; ultrasound; endoscopic surgery; real-time; bur hole; surgical technique; vascular disorders

$\mathrm{H}$ YPERTENSIVE intracerebral hemorrhage (ICH) is a very common neurosurgical condition encountered in clinical practice. Evacuation of the hematoma has recently been performed endoscopically, instead of via open craniotomy, to minimize brain injury. ${ }^{1-4,8}$ However, the 2D visualization of the hematoma that is afforded by an endoscope provides only limited information on intracerebral orientation. Intraoperative ultrasonography is a noninvasive diagnostic $2 \mathrm{D}$ imaging technique that can be used to provide $3 \mathrm{D}$ virtual imaging by moving the probe. The skull disturbs the penetration of ultrasound, but this problem can be resolved by placement of another bur hole. This study was undertaken to evaluate the utility of realtime ultrasound-guided endoscopic surgery for treatment of patients with putaminal hemorrhage.

\section{Methods}

This study was approved by the institutional review board of Yamaguchi University Hospital.

Patients with hypertensive putaminal hemorrhage were prospectively enrolled into the study between November

ABBREVIATIONS GCS = Glasgow Coma Scale; GOS = Glasgow Outcome Scale; ICH = intracerebral hemorrhage .

SUBMITTED June 30, 2014. ACCEPTED November 25, 2014.

INCLUDE WHEN CITING Published online June 5, 2015; DOI: 10.3171/2014.11.JNS141508.

DISCLOSURE The authors report no conflict of interest concerning the materials or methods used in this study or the findings specified in this paper. 
2012 and March 2014. Endoscopic evacuation of the hematoma was performed for the patients who had hematomas $>30 \mathrm{~cm}^{3}$ upon admission. The hematoma volume was estimated from CT scans according to the formula A $\times \mathrm{B} \times \mathrm{C} / 2$. ${ }^{7}$ The $\mathrm{CT}$ slice with the maximum hematoma diameter was selected. The maximum length (A) and its perpendicular length (B) were measured in centimeters. The hematoma evacuation rate was determined as (preoperative hematoma volume - postoperative hematoma volume)/preoperative hematoma volume.

The following clinical data were collected: 1) patient age and sex, 2) side of the putaminal hemorrhage, 3) time from admission to operation, 4) preoperative and postoperative hematoma volume and hematoma evacuation rate $(\%), 5)$ duration of operation, 6) Day 0 and Day 7 Glasgow Coma Scale (GCS) scores, and 7) Glasgow Outcome Scale (GOS) score obtained 90 days after surgery.

\section{Endoscopic and Ultrasonographic Technique}

Endoscopic surgery was performed using a rigid endoscope (Oi-Handy Pro, Karl Storz), and intraoperative ultrasonography was performed using a Prosound $\alpha-7$ system (Hitachi-Aloka Medical) and a 3- to 8-MHz sector probe for the bur hole (Hitachi-Aloka Medical) (Fig. 1). General anesthesia was induced with the patients in the supine position. The endoscope bur hole was placed 10-12 $\mathrm{cm}$ above the nasion and $2.5-4 \mathrm{~cm}$ lateral to midline to achieve the frontal approach, and the ultrasound bur hole was made in the temporal region (Fig. 2). Two linear skin incisions were made at each bur hole. The location of each of the bur holes was adjusted slightly in accordance with the hematoma location. For the ultrasound bur hole, no dural incision was made because the dura permitted penetration of the ultrasound and protected the brain surface from probe damage. The sonographer applied the probe at the ultrasound bur hole to provide navigation guidance for the surgeon. Initially, the hematoma was observed using ultrasound from the endoscope bur hole to determine the direction of the endoscope tract, and the endoscope sheath (Neurosheath 17.5 F; Medikit) was then inserted to the core of the hematoma under ultrasound guidance from the ultrasound bur hole. Throughout the operation, real-time ultrasound was performed via the ultrasound bur hole. Endoscopic evacuation was performed in a dry field. The sonographer informed the surgeon of the location of the endoscope relative to the hematoma. If there was active bleeding from the cavity surface, the bleeding vessel was ablated using monopolar cautery. After sufficient evacuation was achieved, removal of the hematoma was confirmed by using the endoscope on a wet field with artificial cerebrospinal fluid. The extent of hematoma removal was also confirmed using ultrasound.

All study protocols were conducted in accordance with the principles of the Declaration of Helsinki. Informed consent was obtained from each patient or the family. The study was approved by our institutional review board.

\section{Results}

We included 10 patients with hypertensive putaminal hemorrhages that were estimated to be greater than 30

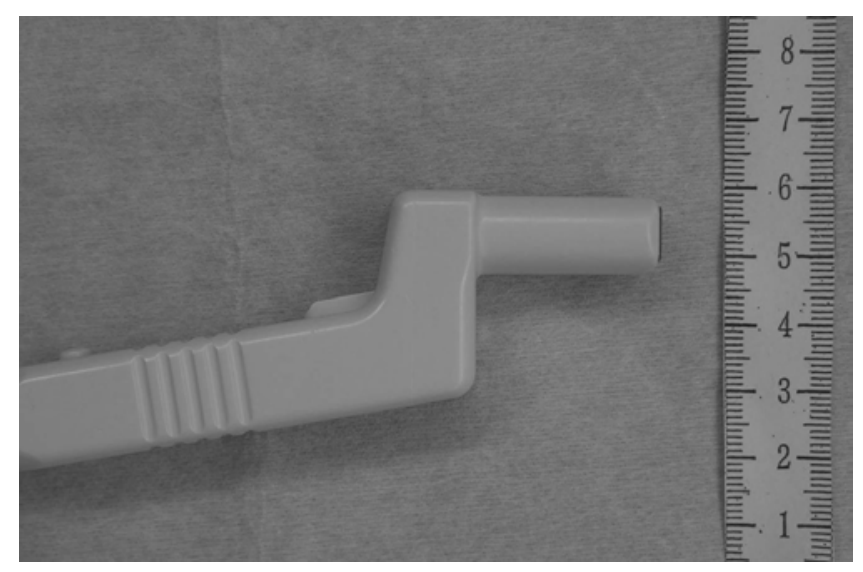

FIG. 1. Photograph of the ultrasound probe that was used in this study (3- to 8-MHz sector probe, Hitachi-Aloka Medical).

$\mathrm{cm}^{3}$ in volume (Table 1). Five of the patients were female and 5 male. The patients' mean age $( \pm$ SD) was $60.9 \pm 8.6$ years. The hemorrhage was on the right side in 8 cases. The mean preoperative hematoma volume was $65.2 \pm 37.1$ $\mathrm{cm}^{3}$, and the mean percentage of hematoma evacuated was $96 \% \pm 3 \%$. Ninety days after the operation, 7 patients were moderately disabled (GOS Score 4), 1 was severely disabled (GOS Score 3), and 2 were in a vegetative state (GOS Score 2). The 2 patients who were in a vegetative state had hematomas volumes greater than $100 \mathrm{~cm}^{3}$ on admission.

\section{Illustrative Case}

Case 10

This 58-year-old woman exhibited hypertensive putaminal hemorrhage with a GCS score of 13. A CT scan showed a right putaminal hemorrhage that was estimated to be $99 \mathrm{~cm}^{3}$ in volume (Fig. 3F). Endoscopic evacuation of the hematoma was performed. The endoscope bur hole was placed $12 \mathrm{~cm}$ above the nasion and $3 \mathrm{~cm}$ lateral to midline, and the ultrasound bur hole was placed at the temporal region. The endoscope sheath was inserted to the center of the hematoma (Fig. 3A), and the hematoma was evacuated under real-time ultrasound guidance in the dry field (Fig. 3B and C). Ultrasound showed that during evacuation with the sheath placed at the center of the hematoma, the residual hematoma moved centripetally. Being able to keep the sheath at the center of the hematoma allowed us to avoid contact with the brain parenchyma and the potential for additional damage. After sufficient evacuation of the hematoma was achieved, a wet field was adopted to confirm the degree of evacuation. The cavity was filled with artificial cerebrospinal fluid, creating a low-echoic space (Fig. 3D), which showed no residual hematoma, and no bleeding was observed in the hematoma cavity with the endoscopic view (Fig. 3E). The postoperative CT scan showed that the hematoma was almost completely evacuated (Fig. 3G).

\section{Discussion}

This is the first report of endoscopic evacuation of ICH- 


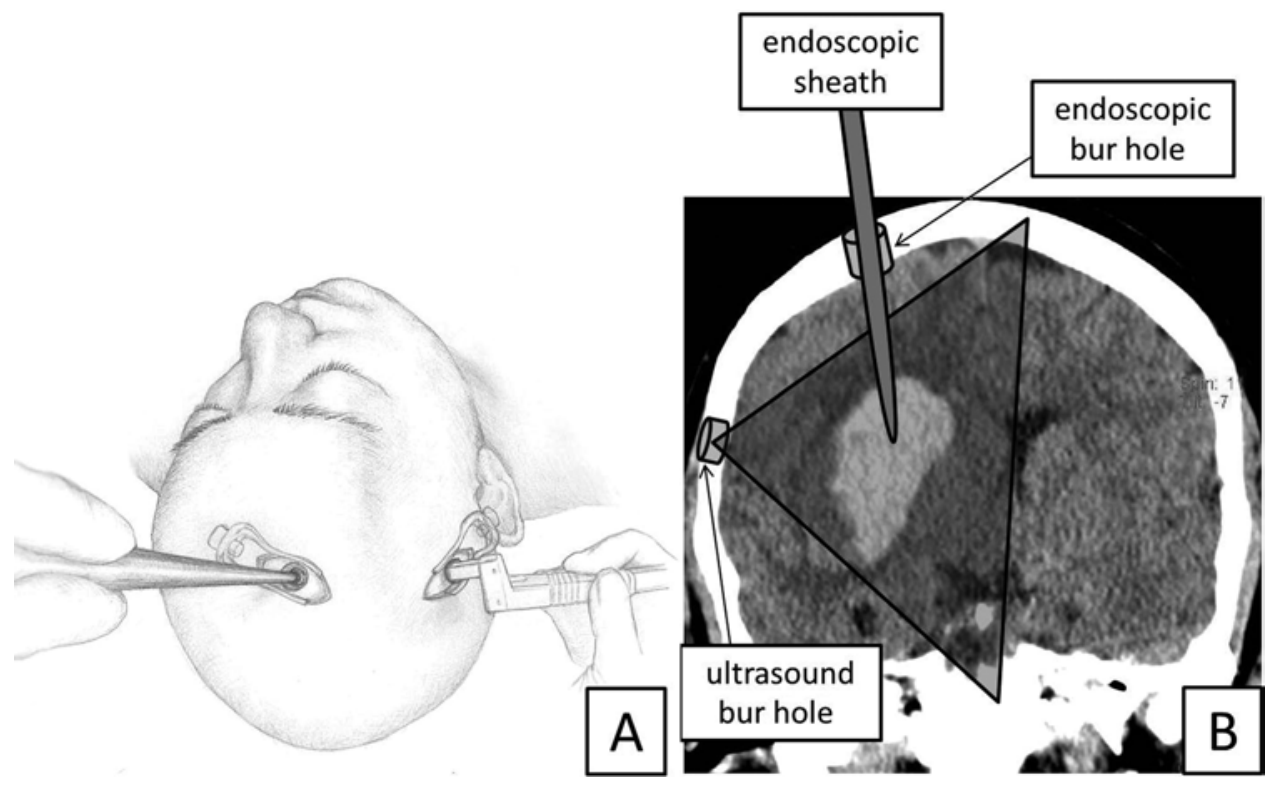

FIG. 2. Illustration and imaging of real-time ultrasound-guided endoscopic surgery. A: Illustration showing the position of the 2 bur holes. The bur hole for the endoscope was located in the vertex, and the bur hole for the ultrasound probe was made in the temporal region. Copyright Mizuya Shinoyama. Published with permission. B: Coronal CT image showing the relationship between the bur holes and the field of view (shaded triangle). An endoscope sheath was inserted through the endoscope bur hole and the hematoma was evacuated under sonographic guidance via the ultrasound bur hole.

related hematomas using real-time ultrasound guidance. Intraoperative navigation with MRI has become widely popular for brain tumor surgery. ${ }^{5,9,10}$ However, MRI navigation is based upon static preoperative information; therefore, intraoperative brain shifting and decrease in hematoma volume cannot be followed. Intraoperative CT or MRI can be used to demonstrate residual hematoma, but these modalities require a break in the surgery and thus cannot be used to confirm the location of the endoscope. Intraoperative ultrasonography performed using the endoscope bur hole is considered useful, but because the probe is applied via the same bur hole as the endoscope, the endoscopic location cannot be observed and the hematoma can be observed only intermittently. In this study, real-time ultrasonography aided in navigation by providing real-time information on the locations of both the endoscope and the residual hematoma. Although our method requires an additional skin incision and bur hole, minimal invasion can be achieved without redundant movement of the sheath or brain injury.

We propose that the sheath should be always placed at the center of the hematoma to avoid brain damage. Kuo et al. recommended that suction be applied via the long axis of the hematoma, because the hematoma could then be evacuated during gradual withdrawal of the sheath. ${ }^{6}$ Their proposal is similar to our own, because they recommended that the sheath should not be moved roughly and that suction should not be applied to the brain, to avoid brain

TABLE 1. Summary of clinical and demographic characteristics of 10 patients with putaminal hemorrhage

\begin{tabular}{|c|c|c|c|c|c|c|c|c|c|c|c|}
\hline \multirow{2}{*}{$\begin{array}{l}\text { Case } \\
\text { No. }\end{array}$} & \multirow{2}{*}{$\begin{array}{l}\text { Age } \\
\text { (yrs) }\end{array}$} & \multirow[b]{2}{*}{ Sex } & \multirow[b]{2}{*}{ Side } & \multirow{2}{*}{$\begin{array}{l}\text { Time to } \\
\text { Op (hrs) }\end{array}$} & \multicolumn{2}{|c|}{ Hematoma Vol $\left(\mathrm{cm}^{3}\right)$} & \multirow{2}{*}{$\begin{array}{c}\text { Hematoma } \\
\text { Evacuation Rate (\%) }\end{array}$} & \multirow{2}{*}{$\begin{array}{l}\text { Duration of Op } \\
\text { (min) }\end{array}$} & \multicolumn{2}{|c|}{ GCS Score } & \multirow{2}{*}{$\begin{array}{l}\text { 90-Day } \\
\text { Outcome }\end{array}$} \\
\hline & & & & & Preop & Postop & & & Day 0 & Day 7 & \\
\hline 1 & 69 & $\mathrm{~F}$ & Right & 1.5 & 50 & 2 & 96 & 130 & 5 & 7 & MD \\
\hline 2 & 72 & $M$ & Right & 1.5 & 127 & 12 & 91 & 120 & 7 & 8 & VS \\
\hline 3 & 54 & M & Right & 2 & 111 & 1 & 99 & 150 & 4 & 4 & VS \\
\hline 4 & 64 & $\mathrm{~F}$ & Left & 1.5 & 31 & 0 & 100 & 90 & 11 & 11 & MD \\
\hline 5 & 65 & $\mathrm{~F}$ & Right & 10 & 32 & 2 & 94 & 110 & 10 & 15 & MD \\
\hline 6 & 55 & M & Right & 2.5 & 39 & 1 & 97 & 110 & 15 & 15 & MD \\
\hline 7 & 67 & M & Left & 1 & 88 & 2 & 98 & 110 & 8 & 10 & SD \\
\hline 8 & 43 & $\mathrm{~F}$ & Right & 20 & 43 & 2 & 95 & 150 & 6 & 9 & MD \\
\hline 9 & 62 & $M$ & Right & 1 & 32 & 2 & 94 & 70 & 13 & 15 & MD \\
\hline 10 & 58 & $\mathrm{~F}$ & Right & 1.5 & 99 & 1 & 99 & 70 & 13 & 15 & MD \\
\hline
\end{tabular}

$\mathrm{MD}=$ moderately disabled (GOS Score 4); SD = severely disabled (GOS Score 3); VS = vegetative state (GOS Score 2). 

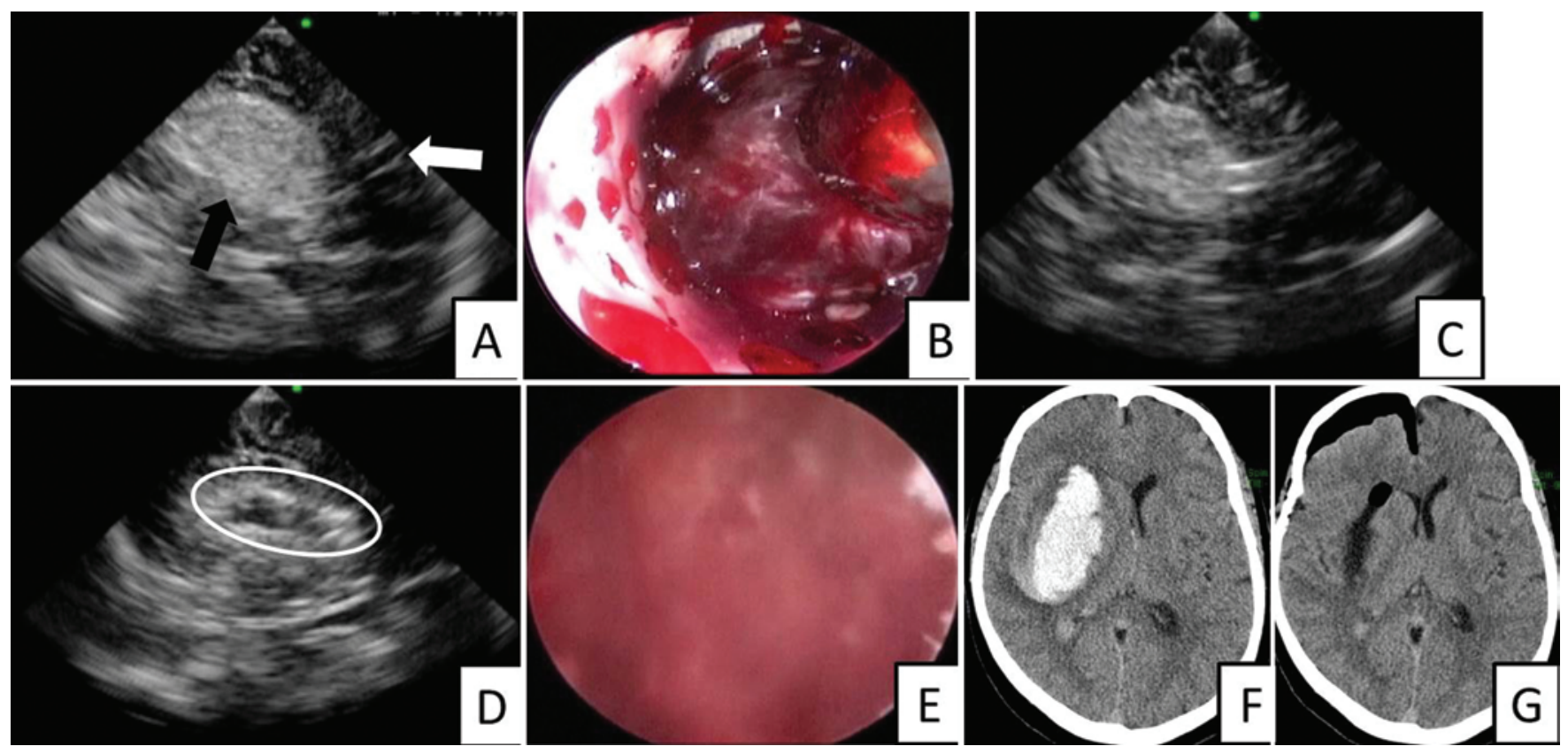

FIG. 3. Real-time ultrasound-guided endoscopic surgery for right putaminal hemorrhage (Case 10). An endoscope sheath (white arrow) was inserted into the center of the hematoma (black arrow) under ultrasound guidance (A), and the hematoma was evacuated with the endoscopic view (B) under guidance (C). The residual cavity was filled with artificial cerebrospinal fluid (wet field) and observed as a low-echoic space (white circle) (D), and the endoscopic view showed no residual hematoma or bleeding (E). The postoperative CT showed no residual hematoma $(\mathbf{G})$. A preoperative CT image $(F)$ is provided for comparison. Figure is available in color online only.

damage or bleeding from the cavity surface. In our study, ultrasound demonstrated that the sheath was inserted to the center of the hematoma; moreover, the intraoperative placement of the sheath was always identified. Evacuation of the central hematoma led to centripetal shrinking of the hematoma, which avoided brain injury. It is very important that real-time navigation allowed for intraoperative compensation of the sheath location, despite brain shift.

In applying this technique, the sonographer must be aware of how to analyze the ultrasound images. Regarding interpretation of the ultrasound findings, the sonographer should work in cooperation with the surgeon, taking the following considerations into account: 1) The endoscope and aspirator are observed as high-echoic objects with acoustic shadows. 2) Intracerebral air can disturb penetration of the ultrasound. 3) When the wall of the hematoma cavity is rough, diffuse reflection of the ultrasound can occur. 4) The sonographer must generate a 3D virtual image from the 2D sonogram to navigate on behalf of the surgeon. Intraoperative ultrasonography is distinct from routine examination because of the use of artificial instruments and air containment; furthermore, the irregular surface of the surgical cavity is quite specific. Despite these complications, when the cavity was filled with artificial cerebrospinal fluid, it was observed as a low-echoic space and the residual hematoma as a higher-echoic space, which could provide simple information regarding the residual hematoma. At present, the ultrasound and endoscope displays are separate; thus, the sonographer and operator cannot watch both displays simultaneously. We propose that in the future, both views should be present in the same display, which might reduce the need for extra time and allow for more cooperation between the surgeon and the sonographer.

There are several problems with and limitations to this study. 1) The endoscope and the application of suction could lead to an acoustic shadow, and it is essential to avoid missing any residual hematoma that is located within the endoscope. 2) The wall of the evacuated hematoma cavity is rough, which could lead to scattering of the ultrasound. In the wet field, the wall was observed as a high, thin, echoic lesion, which could be distinguished from a hematoma based upon the endoscopic view. 3) Evacuation of the hematoma leads to shrinking of the brain; thus, air enters the subdural space, which disturbs the penetration of ultrasound. This problem can, however, be resolved by infusion of artificial cerebrospinal fluid through the endoscope bur hole. 4) This study did not include a control group. Thus, a future randomized study is desirable.

\section{Conclusions}

In endoscopic surgery for putaminal hemorrhage, realtime navigation with ultrasound can demonstrate the location of the endoscope and the residual hematoma, which can thus minimize brain damage. Furthermore, it might decrease brain injury due to the use of an additional bur hole for real-time ultrasonography.

\section{Reference}

1. Chen CC, Cho DY, Chang CS, Chen JT, Lee WY, Lee HC: A 
stainless steel sheath for endoscopic surgery and its application in surgical evacuation of putaminal haemorrhage. J Clin Neurosci 12:937-940, 2005

2. Chen CC, Lin HL, Cho DY: Endoscopic surgery for thalamic hemorrhage: a technical note. Surg Neurol 68:438-442, 2007

3. Chen CC, Liu CL, Tung YN, Lee HC, Chuang HC, Lin SZ, et al: Endoscopic surgery for intraventricular hemorrhage (IVH) caused by thalamic hemorrhage: comparisons of endoscopic surgery and external ventricular drainage (EVD) surgery. World Neurosurg 75:264-268, 2011

4. Hsieh PC, Cho DY, Lee WY, Chen JT: Endoscopic evacuation of putaminal hemorrhage: how to improve the efficiency of hematoma evacuation. Surg Neurol 64:147-153, 2005

5. Kubben PL, ter Meulen KJ, Schijns OE, ter Laak-Poort MP, van Overbeeke JJ, van Santbrink H: Intraoperative MRIguided resection of glioblastoma multiforme: a systematic review. Lancet Oncol 12:1062-1070, 2011

6. Kuo LT, Chen CM, Li CH, Tsai JC, Chiu HC, Liu LC, et al: Early endoscope-assisted hematoma evacuation in patients with supratentorial intracerebral hemorrhage: case selection, surgical technique, and long-term results. Neurosurg Focus 30(4):E9, 2011

7. Kwak R, Kadoya S, Suzuki T: Factors affecting the prognosis in thalamic hemorrhage. Stroke 14:493-500, 1983

8. Nishihara T, Nagata K, Tanaka S, Suzuki Y, Izumi M, Mo- chizuki Y, et al: Newly developed endoscopic instruments for the removal of intracerebral hematoma. Neurocrit Care 2:67-74, 2005

9. Senft C, Franz K, Ulrich CT, Bink A, Szelényi A, Gasser T, et al: Low field intraoperative MRI-guided surgery of gliomas: a single center experience. Clin Neurol Neurosurg 112:237-243, 2010

10. Senft C, Ulrich CT, Seifert V, Gasser T: Intraoperative magnetic resonance imaging in the surgical treatment of cerebral metastases. J Surg Oncol 101:436-441, 2010

\section{Author Contributions}

Conception and design: Sadahiro. Acquisition of data: Sadahiro, Nomura, Goto, Sugimoto, Inamura, Fujiyama, Yamane, Oku. Drafting the article: Sadahiro, Shinoyama. Study supervision: Nomura, Suzuki.

\section{Correspondence}

Hirokazu Sadahiro, Department of Neurosurgery and Clinical Neuroscience, Yamaguchi University School of Medicine, 1-1-1 Minami-Kogushi, Ube, Yamaguchi, Japan. email: sadapiro@ yamaguchi-u.ac.jp. 